\title{
RESEARCH
}

Open Access

\section{Antibiotic-associated dysbiosis affects the ability of the gut microbiota to control intestinal inflammation upon fecal microbiota transplantation in experimental colitis models}

Francesco Strati ${ }^{1 *}$ D, Meritxell Pujolassos ${ }^{2}$, Claudia Burrello ${ }^{1}$, Maria Rita Giuffrè ${ }^{1}$, Georgia Lattanzi ${ }^{1}$, Flavio Caprioli ${ }^{3,4}$, Jacopo Troisi ${ }^{2,5+}$ and Federica Facciotti ${ }^{1 *+}$

\begin{abstract}
Background: The gut microbiota plays a central role in host physiology and in several pathological mechanisms in humans. Antibiotics compromise the composition and functions of the gut microbiota inducing long-lasting detrimental effects on the host. Recent studies suggest that the efficacy of different clinical therapies depends on the action of the gut microbiota. Here, we investigated how different antibiotic treatments affect the ability of the gut microbiota to control intestinal inflammation upon fecal microbiota transplantation in an experimental colitis model and in ex vivo experiments with human intestinal biopsies.

Results: Murine fecal donors were pre-treated with different antibiotics, i.e., vancomycin, streptomycin, and metronidazole before FMT administration to colitic animals. The analysis of the gut microbiome, fecal metabolome, and the immunophenotyping of colonic lamina propria immune cells revealed that antibiotic pre-treatment significantly influences the capability of the microbiota to control intestinal inflammation. Streptomycin and vancomycin-treated microbiota failed to control intestinal inflammation and were characterized by the blooming of pathobionts previously associated with IBD as well as with metabolites related to the presence of oxidative stress and metabolism of simple sugars. On the contrary, the metronidazole-treated microbiota retained its ability to control inflammation co-occurring with the enrichment of Lactobacillus and of innate immune responses involving iNKT cells. Furthermore, ex vivo cultures of human intestinal lamina propria mononuclear cells and iNKT cell clones from IBD patients with vancomycin pre-treated sterile fecal water showed a Th1/Th17 skewing in CD4 ${ }^{+} \mathrm{T}$-cell populations; metronidazole, on the other hand, induced the polarization of iNKT cells toward the production of IL10.

(Continued on next page)
\end{abstract}

\footnotetext{
*Correspondence: francesco.strati@ieo.it; federica.facciotti@ieo.it

${ }^{\dagger} J a c o p o$ Troisi and Federica Facciotti contributed equally to this work.

'Department of Experimental Oncology, IEO European Institute of Oncology IRCCS, Milan, Italy

Full list of author information is available at the end of the article
}

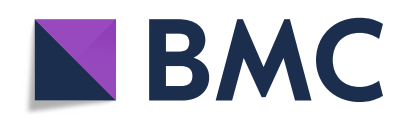

(- The Author(s). 2021 Open Access This article is licensed under a Creative Commons Attribution 4.0 International License, which permits use, sharing, adaptation, distribution and reproduction in any medium or format, as long as you give appropriate credit to the original author(s) and the source, provide a link to the Creative Commons licence, and indicate if changes were made. The images or other third party material in this article are included in the article's Creative Commons licence, unless indicated otherwise in a credit line to the material. If material is not included in the article's Creative Commons licence and your intended use is not permitted by statutory regulation or exceeds the permitted use, you will need to obtain permission directly from the copyright holder. To view a copy of this licence, visit http://creativecommons.org/licenses/by/4.0/. The Creative Commons Public Domain Dedication waiver (http://creativecommons.org/publicdomain/zero/1.0/) applies to the data made available in this article, unless otherwise stated in a credit line to the data. 
(Continued from previous page)

Conclusions: Diverse antibiotic regimens affect the ability of the gut microbiota to control intestinal inflammation

in experimental colitis by altering the microbial community structure and microbiota-derived metabolites.

Keywords: FMT, IBD, Antibiotics, Gut microbiota, iNKT

\section{Background}

The human gut microbiota plays a crucial role in maintaining the integrity of the GI tract, the immune system homeostasis, and the host energy metabolism. Alterations of this network can lead to pathological abnormalities and inflammation [1]. Antibiotics revolutionized medicine regarding the treatment of infectious diseases worldwide; however, their effects are not limited to pathogens for which they are prescribed and indiscriminately affect also the growth of beneficial microbes, including those residing in the gut [2]. By decreasing the diversity of the microbiota, antibiotics compromise host-microbes interactions, immune system homeostasis [2], and impair colonization resistance toward incoming pathogenic bacteria [3]. Indeed, antibiotic treatments worsen the severity of dextran sodium sulfate (DSS)-induced colitis, a mouse model of inflammatory bowel diseases (IBD), through the disturbance of toll-like receptors (TLRs) signaling [4]. Antibiotic-induced dysbiosis promote also sustained $\mathrm{T}$ cell-mediated dysfunctions and increased susceptibility to inflammation and infections by interfering with microbiota-dependent regulation of intestinal innate immunity [5]. Accordingly, we showed that shortterm broad-spectrum oral antibiotic treatment profoundly affects the phenotype and function of mucosal immune cell populations by inducing inflammatory activation of colonic iNKT and $\mathrm{T}$ helper cells [6]. These studies highlight the important implications of repeated broad-spectrum antibiotic use and the breakdown of tolerance mechanisms between the microbiota and the immune system. Strong epidemiological evidences link antibiotic usage early in life with an increased risk of inflammatory conditions such as IBD, indicating an important role for the gut microbiota in modulating intestinal immunity $[7,8]$. It has been proposed that the effects of antibiotics on gut microbiota composition and host physiology may relate to their mechanism of action. For instance, vancomycin, which is a $\mathrm{Gram}^{+}$targeting drug [9] affects also $\mathrm{Gram}^{-}$commensals causing longlasting susceptibility to a variety of secondary infections [10]. On the contrary, low-dose metronidazole is associated with a decreased rate of endoscopic recurrence of Crohn's disease after ileal resection [11].

Presently, little is known about the interaction between the gut microbiota, their metabolites, and relevant inflammatory responses in the gut and the impact on host immunity. We recently showed that control of experimental intestinal inflammation by fecal microbiota transplantation (FMT) is dependent on the transfer of a core microbial ecology composed by different bacterial taxa (Bacteroidales S24-7, Lachnospiraceae, Lactobacillaceae, Ruminococcaceae, Rikenellaceae, Bifidobacteriaceae, and Erysipelotrichaceae) sharing similar metabolic functions (i.e., SCFA production, $\mathrm{Ph}$ control, free radicals scavenging) $[12,13]$. We showed that when this core microbiota is maintained at a certain population level, it creates environmental conditions sufficient to inhibit the growth of pathogenic species/pathobionts, shapes host mucosal immune cell functions, and supports optimal host health [13]. Indeed, transfer of a gut microbiota enriched in these bacterial taxa during acute and chronic experimental colitis is capable to modulate directly both innate and adaptive mucosal immune responses by inducing IL10dependent anti-inflammatory responses [12, 13].

Here, we investigated how antibiotics-conditioned gut microbiota modulates the disease outcome in a model of experimental colitis upon FMT. Amplicon-based 16S rRNA gene analysis of the gut microbiota, fecal metabolomics, and immunophenotyping of colonic lamina propria (LP) immune cells in recipient colitic animals were analyzed. Reciprocal functional interactions between the microbiota, the metabolome, and the mucosal immune system were analyzed to identify key elements fundamental for the restoration of a healthy gut microenvironment and the re-instruction of the immune system toward homeostasis. In vitro/ex vivo experiments in human settings further confirmed our findings.

Taken together, our results shed lights on the effects that alteration of the gut microbiota composition, e.g., by antibiotic treatment, may have on the gut microbiota and its capacity to induce or control intestinal inflammation. These findings may be of pivotal importance in those therapeutic settings in which restoration of homeostatic host-microbes interactions and immune functions are required for the stable management of inflammatory disease.

\section{Methods \\ Mice}

C57BL/6 mice (Charles River, IT) of 8-10 weeks of age were housed at the IEO animal facility in SPF conditions. Experimental groups of mice receiving FMT treatments were kept in separate cages. Littermates of the same sex and age were randomly assigned to the 
different experimental groups. Animal experimentations were approved by the Italian Ministry of Health (Auth. $415 / 2017$ ) and by the animal welfare committee (OPBA) of the European Institute of Oncology (IEO), Italy.

\section{Experimental colitis model and FMT treatments}

For the induction of DSS-mediated acute colitis, mice were given $2 \%(\mathrm{w} / \mathrm{v})$ dextran sodium sulfate (DSS, MW $40 \mathrm{kD}$; TdB Consultancy) in their drinking water for 7 days followed by 2 days of recovery. At sacrifice, colons were collected, their length was measured and divided in portions to isolate lamina propria mononuclear cells (LPMC). FMT was performed through oral gavage of mucus (first day) and feces (second and third days) preparations from donor mice as previously described [13]. Microbiota for FMT was obtained from untreated (normobiotic) mice or mice treated with metronidazole $(1 \mathrm{~g} /$ $\mathrm{L})$, vancomycin $(1 \mathrm{~g} / \mathrm{L})$, or streptomycin $(2 \mathrm{~g} / \mathrm{L})$. Mucus was scraped from colons, diluted in PBS, and administered to recipients at 1:1 ratio. Feces were collected, diluted in PBS $(50 \mathrm{mg} / \mathrm{ml})$, and administered to recipients by oral gavage (10 mg/mouse) 1 day after the end of acute DSS administration to recipient animals.

\section{Inflammation immunoscore}

Clinical (colon length) and immunological (proinflammatory genes expression, $\mathrm{CD} 4^{+} \mathrm{T}$ colonic infiltrate and IL10 protein level) parameters were evaluated in colitic mice undergoing or not FMT treatment. A scoring system has been generated and used to evaluate the inflammation status of mice in 5 independent experiments. Points were recorded according to the following scale: Colon length, $>7 \mathrm{~cm}(0), 6-6.9 \mathrm{~cm}(1),<5.9$ $\mathrm{cm}$ (2); proinflammatory gene expression (Illb and Tnf), <0.0099 (0), 0.01-0.099 (1), >0.099 (2); CD4 ${ }^{+} \mathrm{T}$ cell infiltrate (abs numbers and $\left.\% \mathrm{ki} 67^{+}\right),<5000$ and $<10 \%$ (0), 5000-15000 and 10-20\% (1), > 15000 and > $20 \%$ (2); IL10 protein expression (pg), > 20000 (0), 10000-20000 (1), < 10000 (2).

\section{Murine cell isolation}

Single-cell suspensions were prepared from the colon of C57BL/6 as previously described [13]. LPMCs were isolated from colons upon incubation with $5 \mathrm{mM}$ EDTA at $37^{\circ} \mathrm{C}$ for $30 \mathrm{~min}$, followed by further digestion with collagenase IV and DNase at $37^{\circ} \mathrm{C}$ for $1 \mathrm{~h}$. Cells were then separated with a Percoll gradient. After isolation, some cells were re-stimulated in vitro for $3 \mathrm{~h}$ with PMA/ionomycin in the presence of Brefeldin A for cytokine secretion.

\section{RT-qPCR of tissue mRNA}

Total RNA from colonic tissues was isolated as previously described [13]. cDNAs were generated from $1 \mu \mathrm{g}$ of total RNA with reverse transcription kit (Promega). Gene expression levels were evaluated by qPCR and normalized to Rpl32 gene expression.

16S rRNA gene sequencing and data analysis

DNA extraction, 16S rRNA gene amplification, purification, library preparation, and pair-end sequencing on the Illumina MiSeq platform were performed as described in [13]. Reads were pre-processed using the MICCA pipeline (v.1.5) (http://www.micca.org) [14]. Forward and reverse primers trimming and quality filtering were performed using micca trim and micca filter, respectively. De novo greedy clustering and chimera filtering were performed by using micca otu: operational taxonomic units (OTUs) were assigned by clustering the sequences with a threshold of $97 \%$ pairwise identity, and their representative sequences were taxonomically classified using micca classify with the RDP classifier [15]. Multiple sequence alignment (MSA) of 16S rRNA gene sequences was performed using the Nearest Alignment Space Termination (NAST) [16] algorithm implemented in micca $m s a$ with the template alignment clustered at 97\% similarity of the Greengenes database [17] (release 13_05). Phylogenetic trees were inferred using micca tree [18]. Sampling heterogeneity was reduced rarefying samples at the depth of the less abundant sample using micca tablerare. Alpha (within-sample richness) and beta-diversity (between-sample dissimilarity) estimates were computed using the phyloseq $R$ package [19]. Permutational multivariate analysis of variance (PERM ANOVA) test was performed using the adonis() function in the $\mathrm{R}$ package vegan with 999 permutations. Linear discriminant effect size analysis (LEfSe) was performed to find features (microbial taxa) most likely to explain differences between classes [20]. Random Forest [21] analyses of $16 \mathrm{~S}$ rRNA gene sequencing data were performed using the randomForest $\mathrm{R}$ package; permutation tests with 1000 permutations were performed to assess model significance [22].

\section{Metabolomic analysis}

Metabolome extraction, purification, and derivatization were carried by means of the MetaboPrep kit (Theoreo srl, Montecorvino Pugliano, Salerno, Italy) according to the manufacturer's instruction. Two-microliter samples of the derivatized solution were injected into the GCMS system (GC-2010 Plus gas chromatograph coupled to a 2010 Plus single quadrupole mass spectrometer; Shimadzu Corp., Kyoto, Japan). Chromatographic separation was achieved as previously reported [13, 23] using a $30 \mathrm{~m} 0.25 \mathrm{~mm}$ CP-Sil $8 \mathrm{CB}$ fused silica capillary GC column with $1.00 \mu \mathrm{m}$ film thickness from Agilent (Agilent, J\&W Scientific, Folsom, CA, USA), with helium as carrier gas. Untargeted metabolites were identified by 
comparing the mass spectrum of each peak with the NIST library collection (NIST, Gaithersburg, MD, USA). To identify metabolites identity, the linear index difference max tolerance was set at 10 , while the minimum matching for the NIST library search was set at $85 \%$. According to MSI level 1 standard [24], the relevant putative metabolites identity was further confirmed by means of an independent analytical standard analysis. The normalization procedures consisted of data transformation and scaling. Data transformation was made by generalized log transformation and data scaling by autoscaling (mean-centered and divided by the standard deviation of each variable). To analyze metabolomic data, partial least square discriminant analysis (PLS-DA) [25] was performed on Internal Standard peak area [26] normalized chromatogram using R. Mean centering and unit variance scaling were applied for all analyses. Classification and cross-validation were performed using the wrapper function included in the caret package. A permutation test was performed to assess the significance of class discrimination. Variable importance in projection (VIP) scores were calculated for each component. To identify the most meaningful changes in two conditions, the volcano plot was built by plotting the negative log of the $p$ value on the $y$ axis.

\section{Antibiotic treatment of stool samples and fecal water preparation}

Stool samples from three adult healthy donors were resuspended 1:10 (w/v) in pre-reduced PBS + 10\% FBS and filtered through a $0.75 \mu \mathrm{m}$ filter to remove large debris. The fecal slurries were then incubated with metronidazole $(333 \mu \mathrm{g} / \mathrm{ml})$ streptomycin $(333 \mu \mathrm{g} / \mathrm{ml})$ and vancomycin $(125 \mu \mathrm{g} / \mathrm{ml})$, respectively, at $37^{\circ} \mathrm{C}$ in anaerobiosis for $8 \mathrm{~h}$. After the antibiotic treatments, samples were centrifuged at $13.000 \mathrm{rpm} \times 15^{\prime}$ and sterile filtered through a $0.2-\mu \mathrm{m}$ filter to obtain the sterile fecal water samples. Before fecal water preparation, the antibiotictreated fecal slurries were plated on GAM agar at $37^{\circ} \mathrm{C}$ in anaerobiosis for $72 \mathrm{~h}$ for bacterial cell count.

\section{Ex vivo LPMC stimulation assay}

Human LPMCs were isolated from specimens of IBD patients undergoing intestinal surgical resection as previously described [27]. The study protocol was approved by the ethics committee (Comitato Etico Milano Area 2) of the Fondazione IRCCS Cà Granda, Ospedale Maggiore Policlinico, Milan, Italy (ref \#557_2018). Briefly, the dissected intestinal mucosa was freed of mucus and epithelial cells in sequential steps with DTT $(0.1 \mathrm{mmol} / \mathrm{l})$ and EDTA (1 mmol/l) (both from Sigma-Aldrich) and then digested with collagenase $\mathrm{D}(400 \mathrm{U} / \mathrm{ml})$ (Worthington Biochemical Corporation) for $5 \mathrm{~h}$ at $37^{\circ} \mathrm{C}$. LPMCs were then separated with a Percoll gradient and cultured in complete RPMI 1640 medium containing 5\% human serum (Sigma-Aldrich) and $100 \mathrm{U} / \mathrm{ml} \mathrm{IL-2} \mathrm{(Proleukin).}$ LPMCs were plated at a density of $2 \times 10^{5}$ and treated with the fecal water samples $(1: 5 \mathrm{v} / \mathrm{v}$, fecal water:cells) for $24 \mathrm{~h}$. At the end of the experiment, cells were analyzed with flow cytometry and supernatants were collected for ELISA assays. Cell viability was checked with Zombie Yellow $^{\mathrm{Tm}}$ Fixable Viability Kit (Biolegend).

\section{iNKT cell clones generation and in vitro stimulation}

Human iNKT cell lines were generated from sorted $\mathrm{CD}_{4} 5^{+} \mathrm{CD} 3{ }^{+} \mathrm{CD} 1 \mathrm{~d}:$ PBS57Tet ${ }^{+}$cells isolated from total LPMCs as previously described [28]. Sorted iNKT cells were expanded in vitro for 2 weeks in the presence of irradiated peripheral blood feeders, hIL2 (100 U/ml; Proleukin), and PHA (1 $\mu \mathrm{g} / \mathrm{ml}$; Sigma-Aldrich). iNKT cell clones were generated via cloning by limiting dilution according to the protocol described in [29]. For fecal water stimulation, $5 \times 10^{4}$ THP1 cells were plated in each well in 1:1 ratio with human iNKT cells and treated with the fecal water samples $(1: 5 \mathrm{v} / \mathrm{v}$, fecal water:cells) for $24 \mathrm{~h}$. $\alpha \mathrm{GalCer}$ was used at $100 \mathrm{ng} / \mathrm{ml}$ as control for iNKT cells stimulation.

\section{Flow cytometry}

Human and murine LPMC were stained with labeled antibodies diluted in PBS with $1 \%$ heat-inactivated fetal bovine serum (FBS) for $20 \mathrm{~min}$ on ice. Intracellular staining of cytokines was performed after cell fixation and permeabilization with cytofix/cytoperm (BD) before addition of the antibodies. Samples were analyzed with a FACSCelesta flow cytometer (BD Biosciences, Franklin Lakes NJ, USA). The antibodies and dyes used in the study are reported in Table S1. Data were analyzed using the FlowJo software (TreeStar, Ashland, OR, USA) or FACS Diva software (BD Biosciences, Franklin Lakes NJ, USA).

\section{ELISA assay}

Cytokines from LPMC supernatants were detected by coating with anti-human capturing antibody for IL17, IFNy, TNFa, IL6, and IL10 (Biolegend) overnight at $4{ }^{\circ} \mathrm{C}$. Blocking of the plates was performed with PBS/ BSA $1 \%$ for $1 \mathrm{~h}$ at room temperature, while sample incubation was performed overnight at $4{ }^{\circ} \mathrm{C}$. Standards and samples were analyzed in duplicates. Protein detection was performed with $1 \mu \mathrm{g} / \mathrm{ml}$ of biotin anti-human detection antibody for IL17, IFNy, TNF $\alpha$, IL6, or IL10 (Biolegend) for $2 \mathrm{~h}$ at room temperature. Streptavidin-HRP conjugate (Biolegend) and TMB chromogen solution (ThermoFisher) were used to develop the assay, according to manufacturer's instruction. Reaction was blocked using sulfuric acid $1 \mathrm{~N}$. Plates were read at a wavelength of $450 \mathrm{~nm}$. Murine colonic tissues were homogenized in 

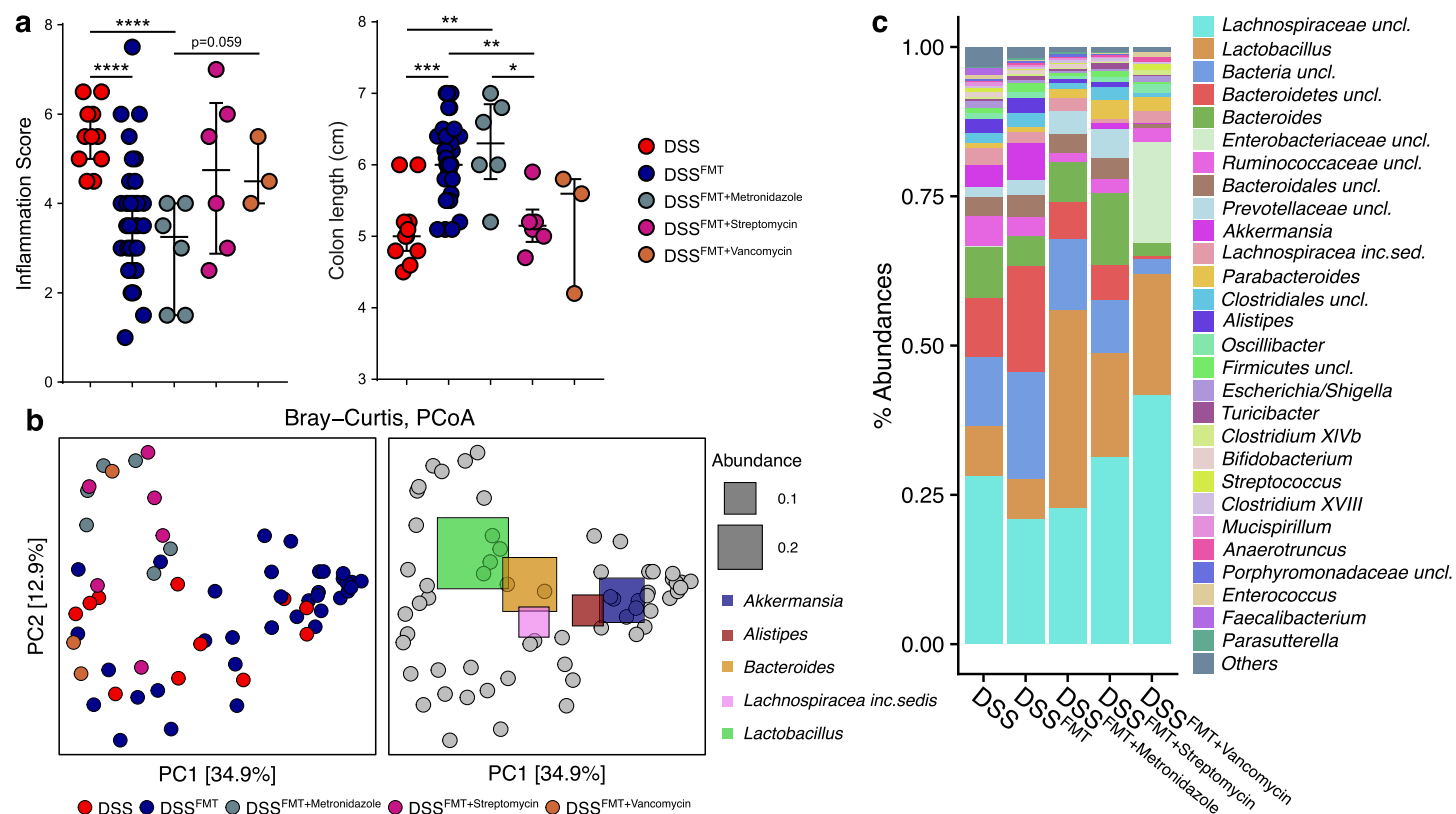

d
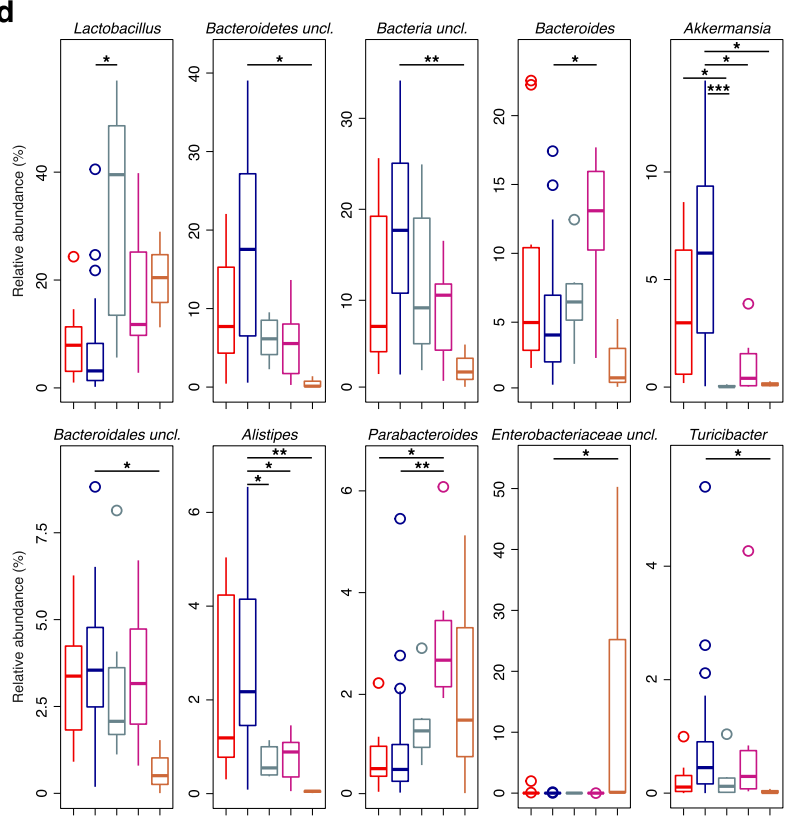

Alistipes
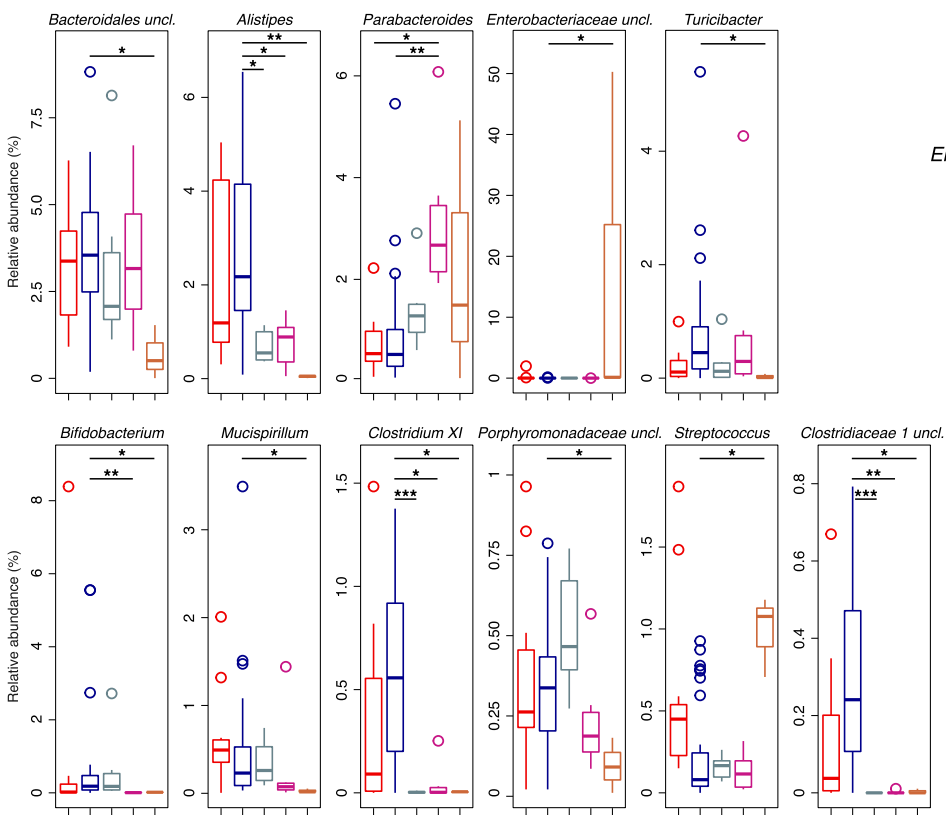

e

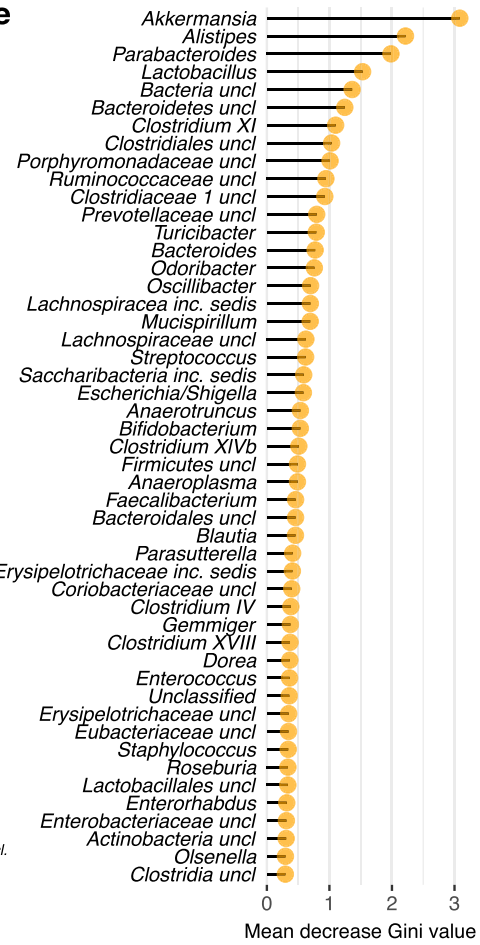

白 DSS

白 DSS FUT

自 DSS FiTT Meronodizale

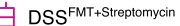

白 $\mathrm{DSS}^{\mathrm{FMT}+\text { Vancomycin }}$

Fig. 1 (See legend on next page.) 
(See figure on previous page.)

Fig. 1 Experimental colitis outcome and gut microbiota composition upon FMT with antibiotics pre-conditioned microbiota in DSS colitic animals. a Experimental colitis outcome as measured by inflammation scores and colon length. b PCoA of beta-diversity as measured by BrayCurtis dissimilarity. In the right panel, PCoA generated as in the left panel showing the five most abundant, fully classified, genera superimposed as colored squares, with size being proportional to the mean relative abundance of the taxon across all samples (in gray). c Mean relative abundance at genus level of the gut microbiota among groups. All genera with relative abundance $<0.1 \%$ are reported together and labeled as "others." $\mathbf{d}$ Most abundant genera (with relative abundance $>0.1 \%$ ) showing significant differences in their relative abundance among groups. $P$ values were FDR corrected. ${ }^{*} p<0.05,{ }^{* *} p<0.01,{ }^{* * *}<0.005$; Wilcoxon rank-sum test. e Random forest analysis. Bacterial taxa with the highest discriminatory power sorted by mean decrease GINI value

$300 \mu$ l RIPA buffer (Cell Signaling Technology) supplemented with phosphatase inhibitors (Sigma) and protease inhibitors (Complete Ultra tablets, Roche). The samples were then incubated at $4{ }^{\circ} \mathrm{C}$ for 30 min under slow rotation and then centrifuged at $13000 \mathrm{rpm}$ for $15 \mathrm{~min}$ at $4{ }^{\circ} \mathrm{C}$. The supernatant was quantified at the NanoDrop with Bradford Assay (BioRad). mIL10 was measured on $6.25 \mu \mathrm{g}$ of lysate using an ELISA assay (Purified anti-mouse IL10 and Biotin anti-mouse IL10, Biolegend) performed following manufacturer's instructions.

\section{Statistical analysis}

Statistical analyses were performed using $\mathrm{R}$ [30] and GraphPad Prism v8.3.1 (GraphPad Software, La Jolla, CA, USA). At least two independent replicates have been performed per each experiment. Data were represented as boxplot, i.e., with the lower and upper hinges corresponding to the first and third quartiles (the 25th and 75 th percentiles). The upper and lower whiskers extend no further than $\pm 1.5 \times \mathrm{IQR}$ from the hinges. Outliers were plotted individually. Otherwise, data were represented as barplot with mean \pm standard error of the mean (SEM). The Wilcoxon rank-sum test was used for the comparison between groups, and the resulting $p$ values were corrected for multiple testing controlling the false discovery rate (FDR) [31] if not stated otherwise. Spearman's correlation tests were computed using the psych $\mathrm{R}$ package [32] and $p$ values were FDR-corrected [31] for multiple comparisons. No data were excluded from analyses.

\section{Results}

Antibiotics pre-conditioning affects the ability of the gut microbiota to control intestinal inflammation upon FMT

The composition of the gut microbiota transferred into colitic recipients influence FMT success rate [33]. To understand the impact of gut microbiota alterations on inflammatory conditions, wild-type SPF murine stool donors were pre-treated with antibiotics having different mechanisms of action, i.e., metronidazole, streptomycin, and vancomycin, before FMT administration to recipient colitic animals. The effect of the treatment on disease outcome was evaluated by using a scoring system accounting for clinical and immunological parameters (see the "Methods" section). Pre-conditioned metronidazole FMT-treated mice (DSS ${ }^{\mathrm{FMT}}+$ Metronidazole mice) showed reduced signs of intestinal inflammation similar to mice treated with normobiotic fecal samples (DSS ${ }^{\mathrm{FMT}}$ mice), as indicated by decreased inflammation score and increased colon length compared to untreated colitic mice (DSS mice) (Fig. 1a). On the contrary, preconditioned streptomycin and vancomycin FMT-treated mice $\left(\mathrm{DSS}^{\mathrm{FMT}}+\right.$ Streptomycin and $\mathrm{DSS}^{\mathrm{FMT}}+$ Vancomycin mice, respectively) showed clear signs of intestinal inflammation, similar to those observed in DSS mice (Fig. 1a). Notably, half of DSS ${ }^{\mathrm{FMT}}+$ Vancomycin animals in the study were sacrificed before the experimental endpoint because of colitis severity. To track the modifications of the gut microbiota induced by the different antibiotic treatments upon FMT in colitic animals, we performed a metataxonomic analysis of FMT recipients. The microbial community structures among groups were significantly different as measured by beta-diversity of BrayCurtis dissimilarity, (PERMANOVA $p<0.05$; Fig. 1b; Table S2) suggesting diverse microbial engraftments after FMT. The relative abundances of various taxa were different among groups (Fig. 1c). We observed higher levels of Akkermansia, unclassified Bacteroidetes, Alistipes, Bifidobacterium, and Clostridia (i.e., Clostridium XI, Clostridiaceae1 uncl.) in DSS ${ }^{\mathrm{FMT}}$ mice. $\mathrm{DSS}^{\mathrm{FMT}}+$ Streptomycin and DSS FMT + Vancomycin animals showed the enrichment of the genera Bacteroides, Parabacteroides, Streptococcus as well as the reduction of other taxa among which Bifidobacterium, Mucispirillum, unclassified Clostridiaceae1, and Clostridium XI. Noteworthy, DSS $^{\mathrm{FMT}}+$ Metronidazole mice, that retained a full capability to control intestinal inflammation compared to DSS ${ }^{\mathrm{FMT}}$ + Streptomycin and DSS ${ }^{\mathrm{FMT}}+$ Vancomycin animals, showed a significant increase of the genus Lactobacillus (Fig. 1d). The gut microbiota was able to predict samples according to treatment among untreated colitic mice, normobiotic FMT-treated, and antibiotic pre-conditioned FMT-treated animals by using a Random Forest classifier. The most important features selected by the classifier were indeed among those differentially abundant among groups (Fig. 1d, e). With an out-of-bag (OOB) 


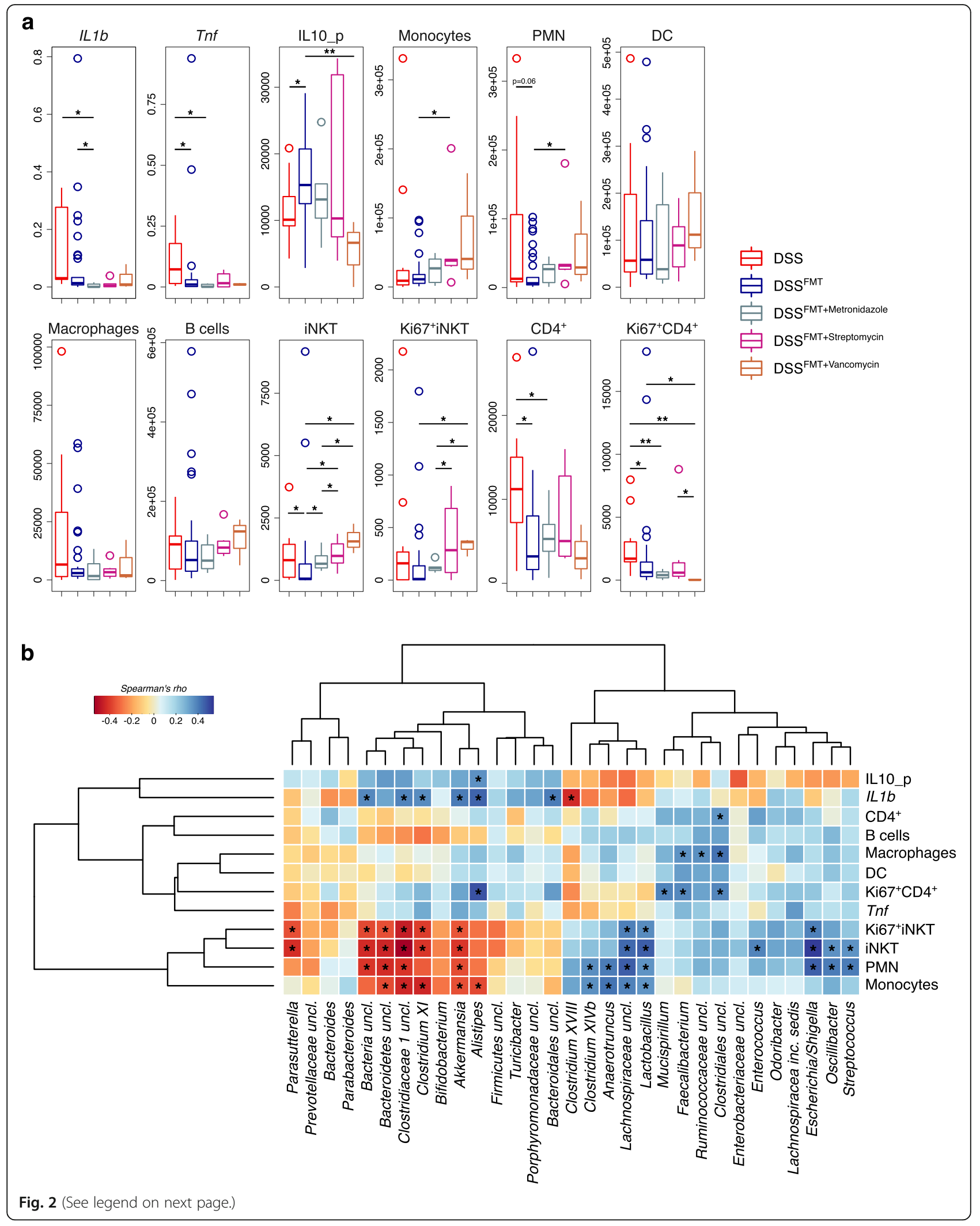


(See figure on previous page.)

Fig. 2 Immunophenotyping and co-occurrence of the gut microbiota with immune cell populations in DSS colitic recipients after FMT with antibiotics pre-conditioned microbiota. a Inflammation markers levels and absolute abundances of innate and adaptive immune cells isolated from the colonic lamina propria of DSS, DSS ${ }^{\mathrm{FMT}}$, DSS ${ }^{\mathrm{FMT}}+$ Metronidazole, DSS ${ }^{\mathrm{FMT}}+$ Streptomycin, and DSS ${ }^{\mathrm{FMT}}+$ Vancomycin ${ }^{\text {animals. }}{ }^{*} p<0.05,{ }^{* *} p<0.01$, *** $<0.001$; Wilcoxon rank-sum test. $\mathbf{b}$ Heatmap of Spearman's $\rho$ ( $\rho s)$ correlations between the relative abundance of the most represented bacterial genera (with relative abundance $>0.1 \%$ ) in the gut microbiota of colitic animals after treatments with the indicated immunological parameters. The significant correlations with an FDR-corrected $p<0.05$ are indicated with an asterisk $\left(^{*}\right)$

error rate of $29.5 \%$, the classifier showed a moderate but significant prediction power (permutation tests $p$ value $=$ 0 , accuracy $=69 \%$, kappa $=43 \%$ ). The classification error was $0.8 \%$ for DSS ${ }^{\mathrm{FMT}}$ and $90 \%$ for DSS samples. These data collectively suggests that FMT is important to stabilize the gut microbiota during experimental colitis despite the inflammatory status of the host.

\section{Transfer of antibiotics preconditioned microbiota in DSS colitic recipients influences colonic iNKT and CD4 ${ }^{+} \mathrm{T}$ cell populations}

The gut microbiota has a profound effect on the intestinal immune system functionality [1]. Therapeutic FMT administered during experimental colitis directly modulates mucosal immunity promoting IL10-dependent antiinflammatory responses $[12,13]$.

Thus, we analyzed the colonic immune cell landscape of FMT-treated animals in relation to the gut microbiota composition (Fig. 2). The presence/absence of specific bacterial taxa in the gut has been linked to the differentiation and expansion of conventional [34] and unconventional $\mathrm{CD}^{+}{ }^{+} \mathrm{T}$ cells [35]. Accordingly, we observed an increase in the numbers of total and proliferating iNKT and $\mathrm{CD} 4^{+} \mathrm{T}$ cells in control colitic animals and FMT recipients treated with a gut microbiota altered by antibiotics preconditioning (Fig. 2a). In particular, DSS mice showed a significant enrichment of $\mathrm{CD} 4^{+}$and $\mathrm{CD} 4^{+} \mathrm{Ki} 67^{+}$ $\mathrm{T}$ cells compared to normobiotic FMT-treated animals. On the other hand, antibiotic preconditioning of the gut microbiota induced the significant increase of LP iNKT cells compared to normobiotic FMT recipients. Furthermore, DSS ${ }^{\mathrm{FMT}}+$ Streptomycin and DSS ${ }^{\mathrm{FMT}+\text { Vancomycin }}$ mice were characterized by the enrichment of proliferating Ki67 ${ }^{+}$iNKT cells compared to $\mathrm{DSS}^{\mathrm{FMT}}$ and $\mathrm{DSS}^{\mathrm{FMT}}+$ Metronidazole animals (Fig. 2a). We then evaluated which bacterial taxa might be important to polarize mucosal immunity toward inflammation by correlating the inflammatory and immunophenotypic data with the gut microbiota (Fig. 2b). The microbial hallmarks of DSS ${ }^{\mathrm{FMT}}$ mice, i.e., Akkermansia, together with Clostridium $X$ and unclassified genera of Bacteroidetes and Clostridiaceae1, negatively correlated with monocytes, PMN, and iNKT cells. On the other hand, different pro-inflammatory taxa such as Streptococcus and Escherichia/Shigella positively correlated with PMN and both iNKT and Ki67 ${ }^{+}$iNKT cells. Also, the genus Lactobacillus correlated with the abundance of LP iNKT cells (Fig. 2b). These data suggest that different members of the gut microbiota modulate the abundance of pro- and anti-inflammatory immune cell populations dictating the success of FMT treatments to control inflammation.

\section{Antibiotics treatments shape the gut microenvironment and microbiota-derived metabolites of DSS colitic recipients}

The phylogenetic composition of the gut microbiota is much more dynamic than community metabolism, as a consequence of microbial functional redundancy [36]. Given the relevant biological implications for host-microbiome interactions, in order to identify microbiota-derived metabolites important to modulate intestinal inflammation, we performed an untargeted metabolomics analysis of fecal samples in colitic animals upon FMT (Fig. 3). We observed significantly different metabolic profiles among groups (Fig. 3a) with azelaic acid, maltose, gluconic acid, norvaline, and erythrose being the metabolites with the highest discriminatory power (Fig. $3 \mathrm{~b})$. Higher levels of azelaic and gluconic acids were detected in $\mathrm{DSS}^{\mathrm{FMT}}+$ Vancomycin mice as well as of erythrose in DSS $^{\mathrm{FMT}}+$ Streptomycin mice (Fig. 3c). The gut metabolome was also able to cluster samples according to their inflammation status (i.e., inflamed vs non-inflamed independently by the treatment) (Figure S1a and b) while the gut microbiota failed to do so (Figure S1c). Nevertheless, we have been able to identify bacterial taxa usually associated with inflammatory conditions and IBD, e.g., Escherichia/Shigella and Fusobacterium in inflamed vs non-inflamed samples (Figure S1d). We then correlated metabolomics and gut microbiota data observing the significant correlation of ferulic acid, gluconate, lactose, and norvaline with different bacterial taxa among which Akkermansia, Bacteroidetes uncl., Clostridium XVIII, Escherichia/Shigella, and Lactobacillus (Fig. 3d). These data suggest that the transfer of different configurations of the gut microbiota affected by antibiotics enrich the gut microenvironment with metabolites that may dictate the status of intestinal inflammation.

\section{Exposure to a vancomycin-conditioned gut} microenvironment skews human $\mathrm{CD}^{+} \mathrm{T}$ cells population toward a Th1/Th17 phenotype while metronidazole promotes IL10 secretion by iNKT cells

Based on our previous data, we hypothesized that the metabolic products of gut microbiota may have an 
a
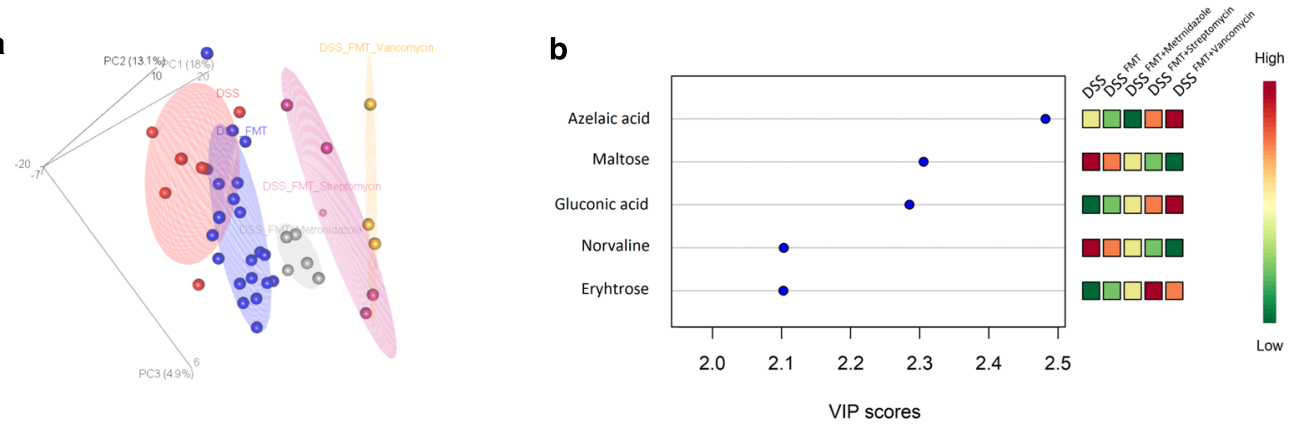

C
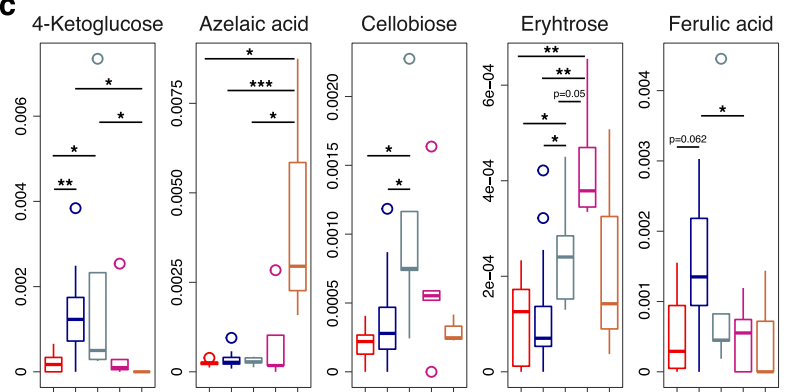

Gentiobiose

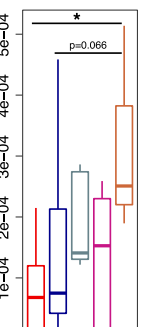

Gluconic acid

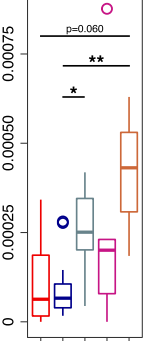

Glucuronic acid Ketoglucose

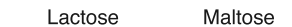

Mannobiose Norvaline
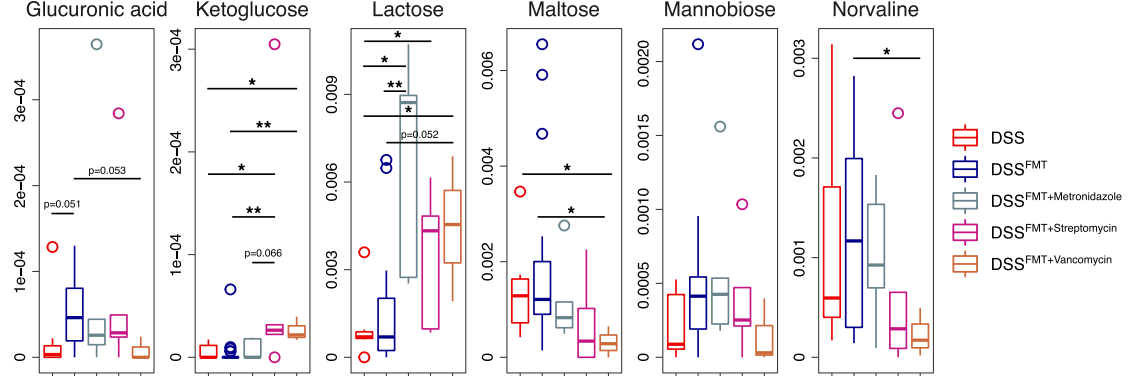

d

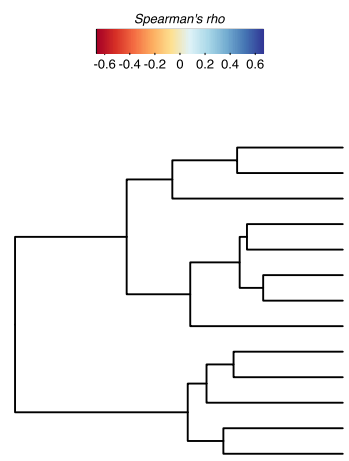

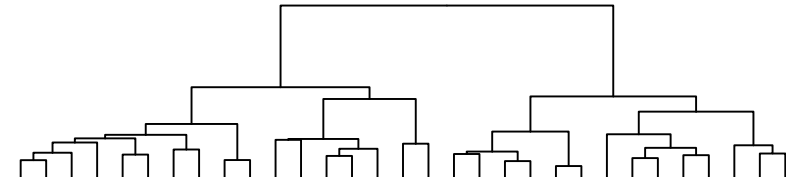

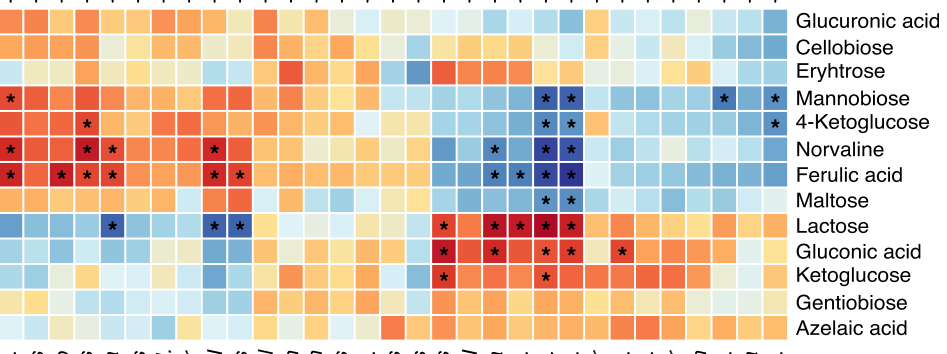

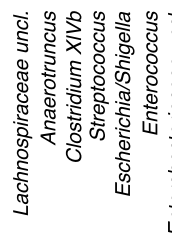
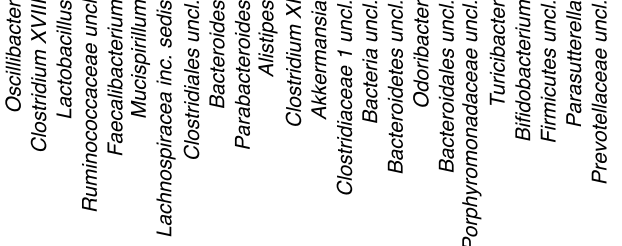

Fig. 3 (See legend on next page.) 
(See figure on previous page.)

Fig. 3 Metabolomics analysis and co-occurrence of the gut microbiota with microbiota-derived metabolites in DSS colitic recipients after FMT with antibiotics pre-conditioned microbiota. a Partial least square discriminant analysis showing clustered samples according to the type of antibiotic treatment. b Metabolites that differentiate samples according to treatment with a variable important in projection (VIP) score $>2$. $\mathbf{c}$ Metabolites with the highest discriminative power for the classification of samples (VIP score $>2$ ) according to treatment. ${ }^{*} p<0.05$, ${ }^{* *} p<0.01$, *** $<0.001$; Wilcoxon rank-sum test. $\mathbf{d}$ Heatmap of Spearman's $\rho$ ( $\rho)$ correlations between the relative abundance of the most represented bacterial genera (with relative abundance $>0.1 \%$ ) in the gut microbiota of colitic animals after treatments with the metabolites with the highest discriminatory power. The significant correlations with an FDR-corrected $p<0.05$ are indicated with an asterisk $\left(^{*}\right)$

important role on the polarization of the host's immune responses to modulate inflammation. Therefore, we performed an ex vivo experiment in which we exposed human colonic lamina propria mononuclear cells (LPMC) of ulcerative colitis patients to sterile fecal water (FW) from antibiotic pre-conditioned human healthy fecal microbiota to mimic the colonic microenvironment of our in vivo model (Fig. 4a). According to our hypothesis, we observed the skewing of $\mathrm{CD} 4^{+} \mathrm{T}$ cells toward a Th1/ Th17 phenotype in the LPMC exposed to the FW from vancomycin-treated microbiota (Fig. 4c and d) as well as significantly higher levels of TNF production compared to metronidazole and untreated (antibiotic-free) FW. TNF levels were significantly increased also upon exposure to streptomycin FW (Fig. 4e). To note, monocytes and APC were not affected by FW treatments. Since iNKT cells were significantly affected by the antibiotics-conditioned microbiota in our in vivo colitis model, we performed an in vitro assay by exposing human intestinal iNKT cell clones isolated from IBD patients [28] to FW. We observed the polarization of iNKT cells toward the production of IL10 upon exposure to the metronidazole-conditioned FW as well as from the untreated FW (Fig. 4b). Interestingly, the microbial composition of the human fecal microbiota after antibiotic exposure (Figure 5a) showed few differences involving principally the genera Bacteroides, Lachnospiracea incertae sedis, Dorea, Oscillibacter, and Peptostreptococcaceae (Fig. 5b). The metabolomic analysis of the FW samples on the contrary showed distinct metabolic profiles according to the type of antibiotic treatment (Fig. 5c) with some highly discriminant metabolites deriving from amino acid catabolism, e.g., glutaric acid, succinate, amino malonate, and urea (Fig. 5d, e). Notably, we observed the significant enrichment of butyric acid in the streptomycinconditioned FW (Fig. 5f, g), a possible indication for reactive oxygen species (ROS) production and accumulation of 8-oxoG lesions [37-39] despite the role of butyrate in regulating homeostatic immune responses $[40,41]$.

\section{Discussion}

Breakdown of the complex interplay between the host immune system and the microbiota increases the risk of pathogen infection, the overgrowth of harmful pathobionts, and inflammatory disease. Although remarkable results have been obtained in understanding the role of the gut microbiota in different human pathologies and on the efficacy of therapies such as FMT and cancer immunotherapy [42, 43], there are paucity of data regarding the mechanism of action for the control of inflammation and the role that different commensals may have on intestinal immune responses. Here, we investigated how modifications of the gut microbiota by different antibiotic treatments affect the disease outcome in a model of acute experimental colitis upon FMT. We further expanded our results paralleling the findings observed in the animal model in a human setting by using colon biopsies from ulcerative colitis patients. We demonstrate that the transfer of an antibiotic-conditioned gut microbiota in colitic animals has an important role for the disease outcome and for the modulation of the inflammatory status, achieved by polarizing the immune response of the FMT recipient and modifying the gut microenvironment. In particular, the colitic recipients transferred with a streptomycin- or vancomycinconditioned microbiota were characterized by blooming of the genera Bacteroides, Parabacteroides, Streptococcus, and unclassified Enterobacteriaceae, all associated with poor outcomes in human clinical studies evaluating FMT efficacy in IBD patients [4446] as well as by the depletion of health-promoting bacteria, i.e., Akkermansia and Bifidobacterium [47, 48]. The gut microenvironment associated with these dysbiotic microbial communities showed the specific enrichment of metabolites that are abundant in the mouse gut after antibiotic treatments leading to susceptibility to Clostridioides difficile infections, i.e., gluconic acid [49], or during steatohepatitis, i.e., azelaic acid [50]. The immunological landscape of the recipients transferred with a vancomycin- or streptomycinconditioned microbiota showed an increased infiltration of proinflammatory polymorphonuclear leukocytes and monocytes as well as proliferating iNKT cells in the intestinal LP. Since colonic APC levels and Ki67 ${ }^{+}$iNKT cells have been shown to contribute to IBD pathogenesis $[6,13$, $28,51,52]$, this could explain the inability of these microbiota configurations to control inflammation. Several reports described also the pivotal role of $\mathrm{T}$ cells as key 


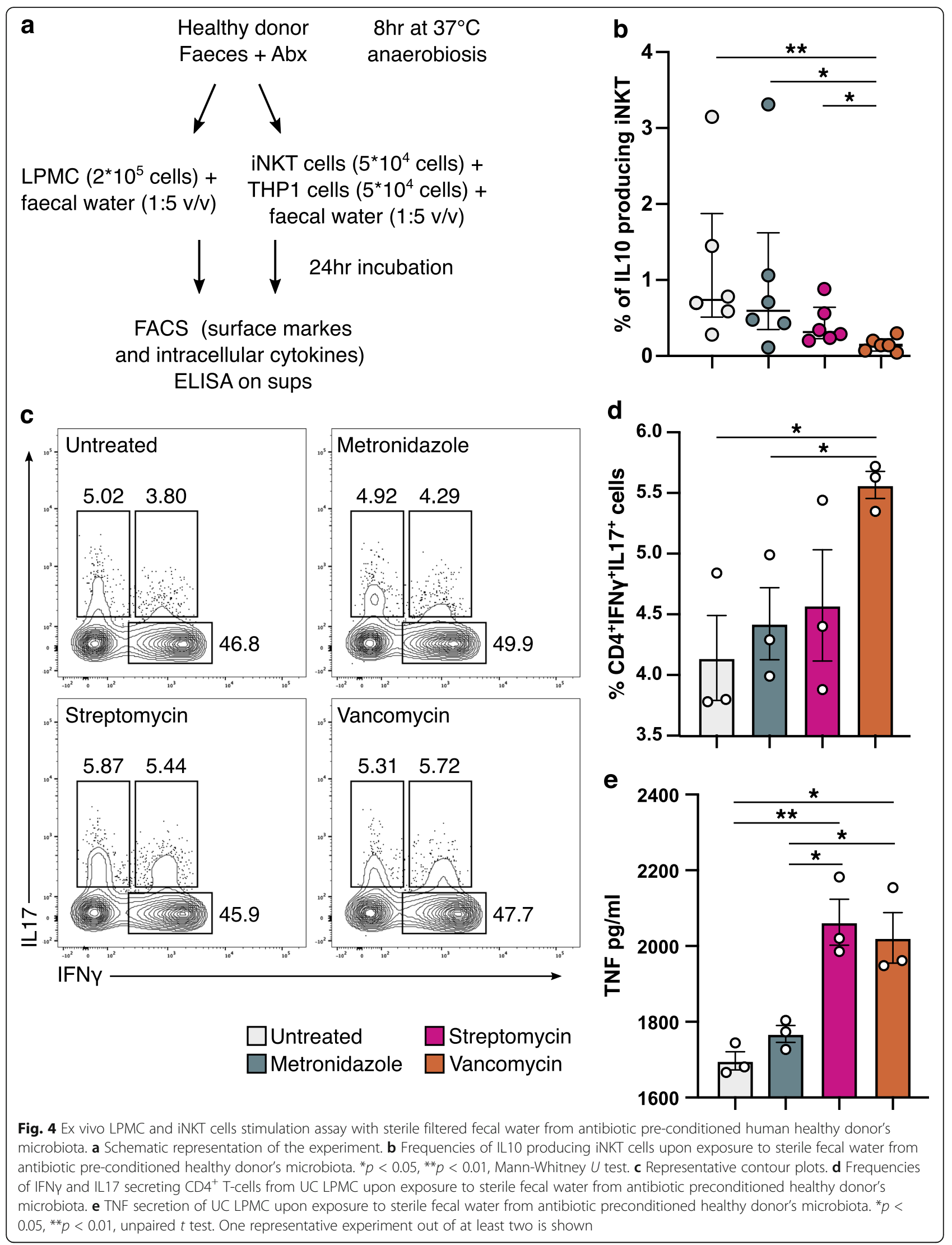




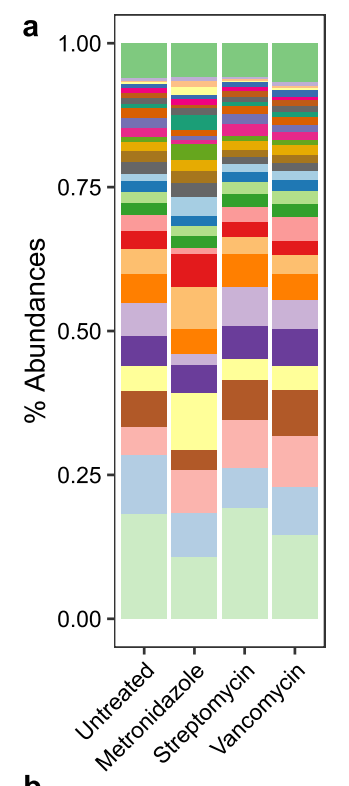

b

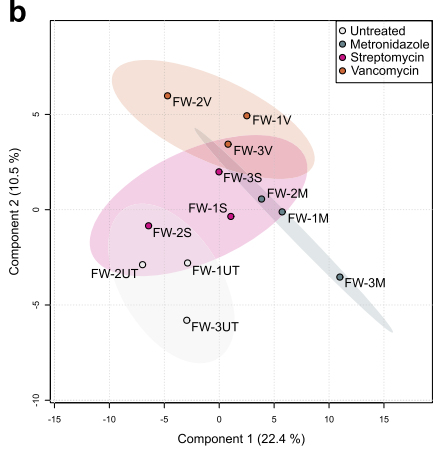

d
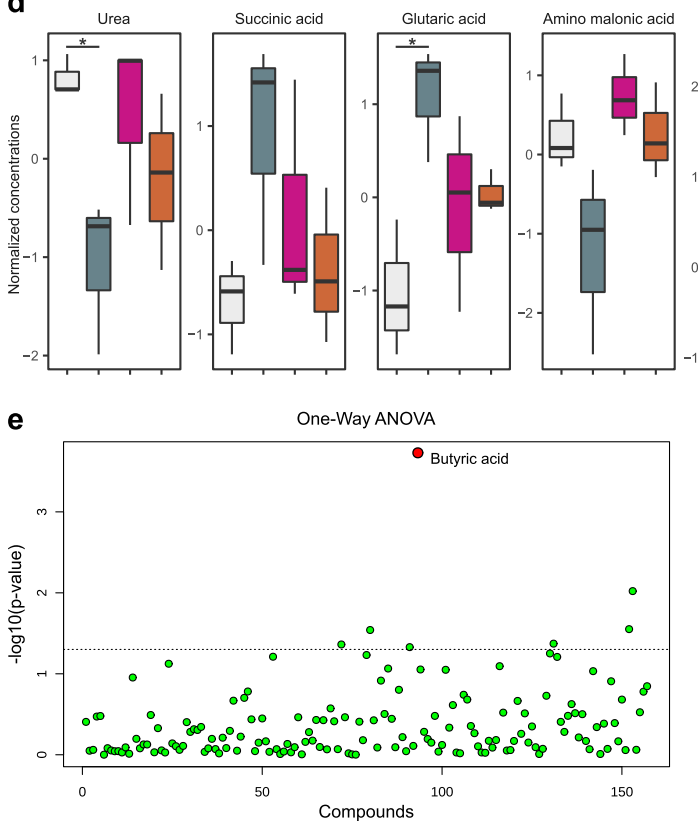

Dorea
Ruminococcus2

Ruminococcus2
Clostridiales uncl.

Anaerostipes

Alistipes

Parabacteroides

Lachnospiraceae uncl. Erysipelotrichaceae uncl.

Clostridium IV

Butyricicoccus

Coprococcus

Bacteria uncl.

Clostridium sensu stricto

Coriobacteriaceae uncl.

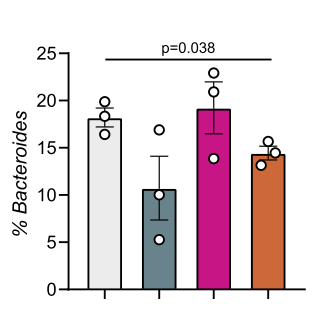

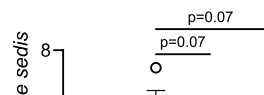

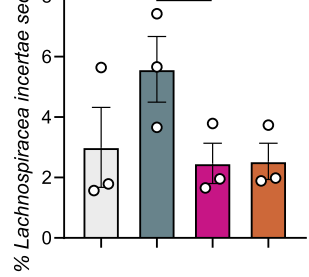

Phascolarctobacterium

Others

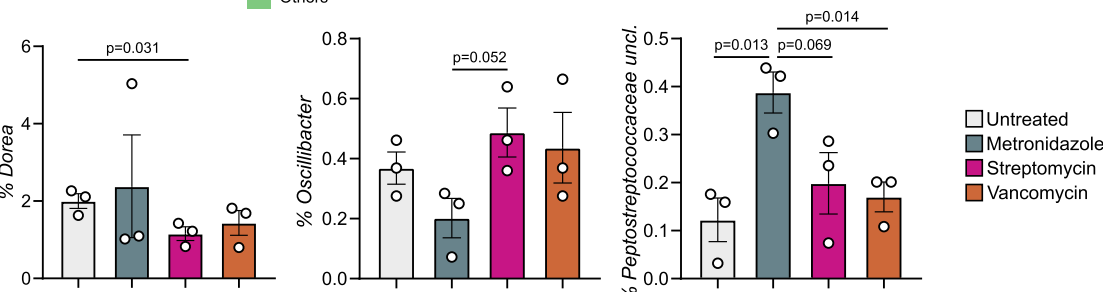

c

C Myristic acid

Glutaric acid

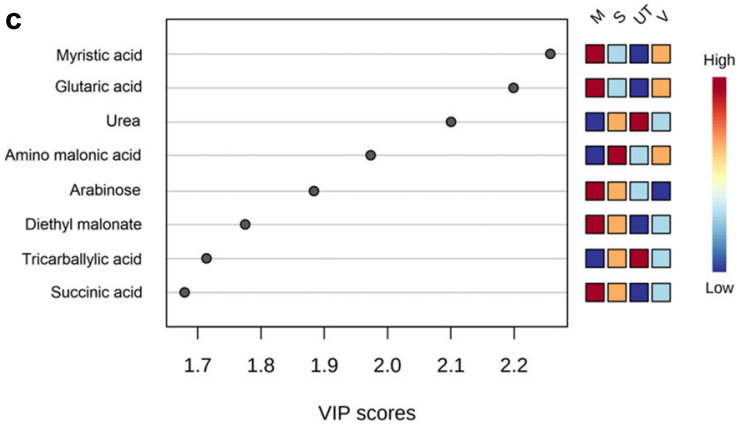

Untreated

Streptomycin ancomycin 
(See figure on previous page.)

Fig. 5 Gut microbiota composition and metabolomics analysis of the antibiotics-conditioned human fecal samples used for the ex vivo experiments. a Mean relative abundance at genus level of the antibiotics-conditioned human gut microbiota. All genera with relative abundance $<0.5 \%$ are reported together and labeled as "others." $\mathbf{b}$ Significantly different taxa identified in the antibiotics-conditioned human gut microbiota. Exact $p$ values are shown; unpaired $t$ test. $\mathbf{c}$ Partial least square discriminant analysis showing clustered fecal water samples according to the type of antibiotic treatment. $\mathbf{d}$ Metabolites that differentiate samples according to treatment by variable important in projection (VIP) scores. e Normalized concentrations of metabolites with the highest discriminative power (VIP score > 1.5) for the classification of samples according to the type of treatment. $\mathbf{f}$ One-way analysis of variance; red dots indicate the significant metabolites $(p<0.05)$ after FDR-correction. $\mathbf{g}$ Normalized concentration of butyric acid as measured in FW samples. ${ }^{*} p<0.05,{ }^{* *} p<0.01,{ }^{* *}<0.001$; unpaired $t$ test

players in the initiation and maintenance of intestinal inflammation through the recognition of bacterial antigens in IBDs [1]. Accordingly, exposure of LPMC from ulcerative colitis patients to vancomycin- and streptomycinconditioned gut microenvironments (i.e., sterile fecal water) enriched with microbiota-derived metabolites polarized $T$ cells toward a proinflammatory Th1/Th17 phenotype and the production of mediators of systemic inflammation, i.e., TNF. This effect could be mediated by the antibiotic-induced alteration of bacterial metabolism, since short-term exposure of healthy human microbiota to antibiotics caused alterations of amino acid catabolic products and enrichment of butyric acid. Although microbiota-derived butyrate is an important mediator of intestinal immune homeostasis $[40,41]$, other studies suggest that the gut microbiota, partially through an altered production of butyrate, induces ROS and the accumulation of 8-oxoG lesions, therefore contributing to inflammation and tumorigenesis [37-39]. On the other hand, the recipients that received FMT with a metronidazoleconditioned microbiota were able to control inflammation by reducing colitis severity. We hypothesize that this effect may be in part mediated by the genus Lactobacillus. Indeed, lactobacilli have a long history of use as healthpromoting bacteria and several studies supported their role in the maintenance of intestinal homeostasis [53, 54]. The recipients receiving the metronidazole-conditioned microbiota showed the enrichment of cellobiose, a metabolite boosting the intestinal levels of Lactobacillus and Bifidobacterium [55]. Nevertheless, cellobiose is also used as a marker of intestinal permeability in Crohn's disease patients [56]. Lactobacillus and Bifidobacterium promotes the release of anti-inflammatory IL10 by APC and T cells, which is pivotal for the resolution of intestinal inflammation [57] by reducing the proliferative capacity of proinflammatory T cells [13]. Also, iNKT cells produce IL10 in murine models of inflammatory disease upon therapeutic FMT [13]. Accordingly, the exposure of human-isolated iNKT cell clones to metronidazole-conditioned fecal water promoted the secretion of IL10, supporting the hypothesis that metronidazole shapes the microbiota and the gut microenvironment favoring a better control of colitis severity and inflammation compared to streptomycin and vancomycin.

\section{Conclusions}

Taken together, our findings confirm that the ability of the gut microbiota to control inflammation is in part mediated by the production of microbiota-derived metabolites. Alterations of the gut microbiota induced by different antibiotic treatments, such as with vancomycin and streptomycin, polarize the immune system toward a pro-inflammatory configuration ablating any benefits of FMT. Metronidazole treatment, on the contrary, allowed the retention of a beneficial microbiota that reduced the severity of colitis suggesting that not all antibiotic treatments are associated with generalized detrimental effects on host-microbes interactions.

\section{Supplementary Information}

The online version contains supplementary material available at https://doi. org/10.1186/s40168-020-00991-x

\begin{abstract}
Additional file 1: Figure S1. Gut Microbiota composition and metabolomics analysis of DSS colitic animals after FMT with antibiotics pre-conditioned microbiota analysed according to their inflammation status. a) Partial Least Square discriminant analysis showing clustered samples according to the inflammation status. b) Metabolites that differentiate samples according to the inflammation status with a Variable Important in Projection (VIP) score > 2. c) Beta-diversity analysis on BrayCurtis dissimilarity ( $p=0.459$, PERMANOVA) d) Most discriminant bacterial taxa identified by LEfSe analysis. Positive and negative LDA scores indicate taxa enriched or depleted in the gut microbiota of samples grouped based on their inflammation status. Only taxa having a $p<0.05$ (Wilcoxon rank-sum test) and LDA $>|2.0|$ are shown.
\end{abstract}

Additional file 2: Table S1. FACS antibodies and dyes.

Additional file 3: Table S2. PERMANOVA of beta-diversity analysis as measured by Bray-Curtis dissimilarity.

Additional file 4: Table S3. Sample metadata, unrarefied OTU table and taxonomic classifications.

\section{Abbreviations \\ ABX: Antibiotics; APC: Antigen-presenting cells; DSS: Dextran sodium sulfate; FMT: Fecal microbiota transplantation; IBD: Inflammatory bowel diseases;} LP: Lamina propria; LPMC: Lamina propria mononuclear cells; Th: T-helper

\section{Acknowledgements}

We would like to thank Erika Mileti and Angélica Díaz-Basabe (IEO) for assistance during the study, the IEO animal facility for the excellent animal husbandry, and Marta Stefanucci (Ospedale Maggiore Policlinico) for the help in collecting human specimens.

\section{Authors' contributions}

$C B, F S, J T$, and FF designed the experiments. MP, MRG, GL, CB, and FS performed the experiments. JT and FS analyzed the data. FC recruited subjects and provided reagents. FS wrote the manuscript. FF conceived the 
study and revised the manuscript. All the authors critically reviewed and approved the manuscript.

\section{Funding}

This work was financially supported by the Italian Ministry of Health (IMMUBAC, GR-2016-02361741) and by AIRC-Investigator Grant 201922923 to FF. The funders had no role in study design, data collection and analysis, decision to publish, or preparation of the manuscript.

\section{Availability of data and materials}

$16 \mathrm{~S}$ rRNA gene sequencing raw data are available in SRA Repository with accession number PRJNA494680 and in the European Nucleotide Archive with accession number PRJEB41434. Sample metadata, unrarefied OTU table, and taxonomic classifications are available in the Supplementary Table S3.

\section{Ethics approval and consent to participate}

Animal experimentations were approved by Italy's Ministry of Health (Auth. 415/2017) and by the animal welfare committee (OPBA) of the European Institute of Oncology (IEO), Italy.

The human study was approved by the ethics committee (Comitato Etico Milano Area 2) of the Fondazione IRCCS Cà Granda, Ospedale Maggiore Policlinico, Milan, Italy (nr. 557_2018).

\section{Competing interests}

The authors declare no competing interests.

\section{Author details}

${ }^{1}$ Department of Experimental Oncology, IEO European Institute of Oncology IRCCS, Milan, Italy. ${ }^{2}$ Theoreo srl, Spin-off Company of the University of Salerno, Montecorvino Pugliano, Italy. ${ }^{3}$ Department of Pathophysiology and Transplantation, Università degli Studi di Milano, Milan, Italy.

${ }^{4}$ Gastroenterology and Endoscopy Unit, Fondazione IRCCS Cà Granda, Ospedale Maggiore Policlinico, Milan, Italy. ${ }^{5}$ European Biomedical Research Institute of Salerno (EBRIS), Salerno, Italy.

Received: 4 August 2020 Accepted: 29 December 2020

Published online: 06 February 2021

\section{References}

1. Kamada N, Seo SU, Chen GY, Nunez G. Role of the gut microbiota in immunity and inflammatory disease. Nat Rev Immunol. 2013;13(5):321-35.

2. Modi SR, Collins JJ, Relman DA. Antibiotics and the gut microbiota. J Clin Invest. 2014;124(10):4212-8.

3. Baumler AJ, Sperandio V. Interactions between the microbiota and pathogenic bacteria in the gut. Nature. 2016;535(7610):85-93.

4. Rakoff-Nahoum S, Paglino J, Eslami-Varzaneh F, Edberg S, Medzhitov R. Recognition of commensal microflora by toll-like receptors is required for intestinal homeostasis. Cell. 2004;118(2):229-41.

5. Scott NA, Andrusaite A, Andersen P, Lawson M, Alcon-Giner C, Leclaire C, Caim S, Le Gall G, Shaw T, Connolly JPR, et al. Antibiotics induce sustained dysregulation of intestinal $T$ cell immunity by perturbing macrophage homeostasis. Sci Transl Med. 2018;10(464):eaao4755.

6. Burrello C, Garavaglia F, Cribiu FM, Ercoli G, Bosari S, Caprioli F, Facciotti F. Short-term oral antibiotics treatment promotes inflammatory activation of colonic invariant natural killer T and conventional CD4(+) T cells. Front Med (Lausanne). 2018;5:21.

7. Kronman MP, Zaoutis TE, Haynes K, Feng R, Coffin SE. Antibiotic exposure and IBD development among children: a population-based cohort study. Pediatrics. 2012:130(4):e794-803.

8. Shaw SY, Blanchard JF, Bernstein CN. Association between the use of antibiotics in the first year of life and pediatric inflammatory bowel disease. Am J Gastroenterol. 2010;105(12):2687-92.

9. Ubeda C, Taur Y, Jenq RR, Equinda MJ, Son T, Samstein M, Viale A, Socci ND, van den Brink MR, Kamboj M, et al. Vancomycin-resistant Enterococcus domination of intestinal microbiota is enabled by antibiotic treatment in mice and precedes bloodstream invasion in humans. J Clin Invest. 2010; 120(12):4332-41.

10. Lewis BB, Buffie CG, Carter RA, Leiner I, Toussaint NC, Miller LC, Gobourne A, Ling L, Pamer EG. Loss of microbiota-mediated colonization resistance to clostridium difficile infection with oral vancomycin compared with metronidazole. J Infect Dis. 2015;212(10):1656-65.
11. Glick LR, Sossenheimer PH, Ollech JE, Cohen RD, Hyman NH, Hurst RD, Rubin DT. Low-dose metronidazole is associated with a decreased rate of endoscopic recurrence of Crohn's disease after ileal resection: a retrospective cohort study. J Crohns Colitis. 2019;13(9):1158-62.

12. Burrello C, Giuffre MR, Macandog AD, Diaz-Basabe A, Cribiu FM, Lopez G, Borgo F, Nezi L, Caprioli F, Vecchi M, et al. Fecal microbiota transplantation controls murine chronic intestinal inflammation by modulating immune cell functions and gut microbiota composition. Cells. 2019;8(6):517.

13. Burrello C, Garavaglia F, Cribiu FM, Ercoli G, Lopez G, Troisi J, Colucci A, Guglietta S, Carloni S, Guglielmetti S, et al. Therapeutic faecal microbiota transplantation controls intestinal inflammation through IL10 secretion by immune cells. Nat Commun. 2018;9(1):5184.

14. Albanese D, Fontana P, De Filippo C, Cavalieri D, Donati C. MICCA: a complete and accurate software for taxonomic profiling of metagenomic data. Sci Rep. 2015;5:9743.

15. Wang Q, Garrity GM, Tiedje JM, Cole JR. Naive Bayesian classifier for rapid assignment of rRNA sequences into the new bacterial taxonomy. Appl Environ Microbiol. 2007;73(16):5261-7.

16. DeSantis T, Hugenholtz P, Keller K, Brodie E, Larsen N, Piceno Y, Phan R, Andersen GL. NAST: a multiple sequence alignment server for comparative analysis of 16S rRNA genes. Nucleic Acids Res. 2006;34(suppl 2):W394-9.

17. DeSantis TZ, Hugenholtz P, Larsen N, Rojas M, Brodie EL, Keller K, Huber T, Dalevi D, Hu P, Andersen GL. Greengenes, a chimera-checked 16S rRNA gene database and workbench compatible with ARB. Appl Environ Microbiol. 2006;72(7):5069-72.

18. Price MN, Dehal PS, Arkin AP. FastTree 2--approximately maximumlikelihood trees for large alignments. PLoS One. 2010;5(3):e9490.

19. McMurdie PJ, Holmes S. Phyloseq: an R package for reproducible interactive analysis and graphics of microbiome census data. PLoS One. 2013;8(4): e61217.

20. Segata N, Izard J, Waldron L, Gevers D, Miropolsky L, Garrett WS, Huttenhower C. Metagenomic biomarker discovery and explanation. Genome Biol. 2011;12(6):R60.

21. Breiman L. Random Forests. Machine Learning. 2001;45(1):5-32.

22. Murphy MA, Evans JS, Storfer A. Quantifying Bufo boreas connectivity in Yellowstone National Park with landscape genetics. Ecology. 2010;91(1):25261.

23. Roggero $P$, Liotto N, Pozzi C, Braga D, Troisi J, Menis C, Gianni ML, Berni Canani R, Paparo L, Nocerino R, et al. Analysis of immune, microbiota and metabolome maturation in infants in a clinical trial of Lactobacillus paracasei CBA L74-fermented formula. Nat Commun. 2020;11(1):2703.

24. Sumner LW, Amberg A, Barrett D, Beale MH, Beger R, Daykin CA, Fan TW, Fiehn O, Goodacre R, Griffin $\mathrm{J}$, et al. Proposed minimum reporting standards for chemical analysis Chemical Analysis Working Group (CAWG) Metabolomics Standards Initiative (MSI). Metabolomics. 2007;3(3):211-21.

25. Wold S, Sjöström M, Eriksson L. PLS-regression: a basic tool of chemometrics. PLS Methods. 2001;58:109-30.

26. Sysi-Aho M, Katajamaa M, Yetukuri L, Oresic M. Normalization method for metabolomics data using optimal selection of multiple internal standards. BMC bioinformatics. 2007;8:93.

27. Caprioli F, Pallone F, Monteleone G. Th17 immune response in IBD: A new pathogenic mechanism. J Crohns Colitis. 2008;2(4):291-5.

28. Burrello C, Pellegrino G, Giuffre MR, Lovati G, Magagna I, Bertocchi A, Cribiu FM, Boggio F, Botti F, Trombetta E, et al. Mucosa-associated microbiota drives pathogenic functions in IBD-derived intestinal iNKT cells. Life Sci Alliance. 2019;2(1):e201800229.

29. Mariotti S, Nisini R: Generation of human T cell clones. In: T Cell Protocols. edn.: Springer; 2009: 65-93.

30. Team RC: A language and environment for statistical computing. $R$ Foundation for statistical computing, 2015; Vienna, Austria. In.; 2016.

31. Benjamini $Y$, Hochberg Y. Controlling the false discovery rate: a practical and powerful approach to multiple testing. J R Stat Soc B Methodol. 1995: 289-300.

32. Revelle W. Psych: procedures for psychological, psychometric, and personality research. R package version 1.3. 10. Evanston: Northwestern University; 2013.

33. Kump P, Wurm P, Grochenig HP, Wenzl H, Petritsch W, Halwachs B, Wagner M, Stadlbauer $V$, Eherer A, Hoffmann KM, et al. The taxonomic composition of the donor intestinal microbiota is a major factor influencing the efficacy of faecal microbiota transplantation in therapy refractory ulcerative colitis. Aliment Pharmacol Ther. 2018;47(1):67-77. 
34. Ivanov II, Atarashi K, Manel N, Brodie EL, Shima T, Karaoz U, Wei D, Goldfarb KC, Santee CA, Lynch SV. Induction of intestinal Th17 cells by segmented filamentous bacteria. Cell. 2009;139(3):485-98.

35. An D, Oh SF, Olszak T, Neves JF, Avci FY, Erturk-Hasdemir D, Lu X, Zeissig S, Blumberg RS, Kasper DL. Sphingolipids from a symbiotic microbe regulate homeostasis of host intestinal natural killer T cells. Cell. 2014;156(1-2):12333.

36. Human Microbiome Project C. Structure, function and diversity of the healthy human microbiome. Nature. 2012;486(7402):207-14.

37. Irrazabal T, Thakur BK, Kang M, Malaise Y, Streutker C, Wong EOY, Copeland J, Gryfe R, Guttman DS, Navarre WW, et al. Limiting oxidative DNA damage reduces microbe-induced colitis-associated colorectal cancer. Nat Commun. 2020;11(1):1802

38. Belcheva A, Irrazabal T, Robertson SJ, Streutker C, Maughan H, Rubino S, Moriyama EH, Copeland JK, Surendra A, Kumar S, et al. Gut microbial metabolism drives transformation of $\mathrm{MSH} 2$-deficient colon epithelial cells. Cell. 2014;158(2):288-99.

39. Kumar A, Wu H, Collier-Hyams LS, Kwon YM, Hanson JM, Neish AS. The bacterial fermentation product butyrate influences epithelial signaling via reactive oxygen species-mediated changes in cullin-1 neddylation. J Immunol. 2009:182(1):538-46.

40. Smith PM, Howitt MR, Panikov N, Michaud M, Gallini CA, Bohlooly YM, Glickman JN, Garrett WS. The microbial metabolites, short-chain fatty acids, regulate colonic Treg cell homeostasis. Science. 2013;341(6145):569-73.

41. Furusawa Y, Obata Y, Fukuda S, Endo TA, Nakato G, Takahashi D, Nakanishi Y, Uetake C, Kato K, Kato T, et al. Commensal microbe-derived butyrate induces the differentiation of colonic regulatory T cells. Nature. 2013; 504(7480):446-50

42. Amoroso C, Perillo F, Strati F, Fantini M, Caprioli F, Facciotti F. The role of gut microbiota biomodulators on mucosal immunity and intestinal inflammation. Cells. 2020;9(5):1234.

43. Perillo F, Amoroso C, Strati F, Giuffre MR, Diaz-Basabe A, Lattanzi G, Facciotti F. Gut microbiota manipulation as a tool for colorectal cancer management: recent advances in its use for therapeutic purposes. Int J Mol Sci. 2020; 21(15):5389.

44. Gao X, Cao Q, Cheng Y, Zhao D, Wang Z, Yang H, Wu Q, You L, Wang Y, Lin $Y$, et al. Chronic stress promotes colitis by disturbing the gut microbiota and triggering immune system response. Proc Natl Acad Sci U S A. 2018; 115(13):E2960-9.

45. Lloyd-Price J, Arze C, Ananthakrishnan AN, Schirmer M, Avila-Pacheco J, Poon TW, Andrews E, Ajami NJ, Bonham KS, Brislawn CJ, et al. Multi-omics of the gut microbial ecosystem in inflammatory bowel diseases. Nature. 2019:569(7758):655-62.

46. Sarrabayrouse G, Landolfi S, Pozuelo M, Willamil J, Varela E, Clark A, Campos D, Herrera C, Santiago A, Machiels K, et al. Mucosal microbial load in Crohn's disease: a potential predictor of response to faecal microbiota transplantation. EBioMedicine. 2020;51:102611.

47. Belzer C, de Vos WM. Microbes inside--from diversity to function: the case of Akkermansia. ISME J. 2012;6(8):1449-58.

48. Ventura M, Turroni F, Motherway MOC, MacSharry J, van Sinderen D. Hostmicrobe interactions that facilitate gut colonization by commensal bifidobacteria. Trends Microbiol. 2012;20(10):467-76.

49. Theriot CM, Koenigsknecht MJ, Carlson PE Jr, Hatton GE, Nelson AM, Li B, Huffnagle GB, ZL J, Young VB. Antibiotic-induced shifts in the mouse gut microbiome and metabolome increase susceptibility to Clostridium difficile infection. Nat Commun. 2014;5:3114.

50. Matsubara T, Tanaka N, Krausz KW, Manna SK, Kang DW, Anderson ER, Luecke H, Patterson AD, Shah YM, Gonzalez FJ. Metabolomics identifies an inflammatory cascade involved in dioxin- and diet-induced steatohepatitis. Cell Metab. 2012;16(5):634-44.

51. Fais S, Pallone F, Squarcia O, Biancone L, Ricci F, Paoluzi P, Boirivant M: HLADR antigens on colonic epithelial cells in inflammatory bowel disease: I. Relation to the state of activation of lamina propria lymphocytes and to the epithelial expression of other surface markers. Clin Exp Immunol 1987, 68(3): 605.

52. Fournier $B$, Parkos $C$. The role of neutrophils during intestinal inflammation. Mucosal Immunol. 2012;5(4):354-66.

53. Hill C, Guarner F, Reid G, Gibson GR, Merenstein DJ, Pot B, Morelli L, Canani RB, Flint HJ, Salminen S. The International Scientific Association for Probiotics and Prebiotics consensus statement on the scope and appropriate use of the term probiotic. Nat Rev Gastroenterol Hepatol. 2014; 11(8):506-14.

54. Marco ML, Heeney D, Binda S, Cifelli CJ, Cotter PD, Foligné B, Gänzle M, Kort R, Pasin G, Pihlanto A. Health benefits of fermented foods: microbiota and beyond. Curr Opin Biotechnol. 2017;44:94-102.

55. van Zanten GC, Krych L, Roytio H, Forssten S, Lahtinen SJ, Abu Al-Soud W, Sorensen S, Svensson B, Jespersen L, Jakobsen M. Synbiotic Lactobacillus acidophilus NCFM and cellobiose does not affect human gut bacterial diversity but increases abundance of lactobacilli, bifidobacteria and branched-chain fatty acids: a randomized, double-blinded cross-over trial. FEMS Microbiol Ecol. 2014;90(1):225-36.

56. Welcker K, Martin A, Kolle P, Siebeck M, Gross M. Increased intestinal permeability in patients with inflammatory bowel disease. Eur J Med Res. 2004;9(10):456-60.

57. Di Giacinto C, Marinaro M, Sanchez M, Strober W, Boirivant M. Probiotics ameliorate recurrent Th1-mediated murine colitis by inducing IL-10 and IL-10dependent TGF- $\beta$-bearing regulatory cells. J Immunol. 2005;174(6):3237-46.

\section{Publisher's Note}

Springer Nature remains neutral with regard to jurisdictional claims in published maps and institutional affiliations.
Ready to submit your research? Choose BMC and benefit from:

- fast, convenient online submission

- thorough peer review by experienced researchers in your field

- rapid publication on acceptance

- support for research data, including large and complex data types

- gold Open Access which fosters wider collaboration and increased citations

- maximum visibility for your research: over $100 \mathrm{M}$ website views per year

At BMC, research is always in progress.

Learn more biomedcentral.com/submissions 